\title{
Special Issues -International Symposium on Polymer Crystallization 2007- Melt Crystallization of isotactic Polybutene-1Trigonal Form: the Effect of Side Chain Entropy on Crystal Growth Kinetics
}

\author{
By Motoi YAMASHITA* and Takuya TAKAHASHI
}

\begin{abstract}
The morphology and crystal growth kinetics of isotactic polybutene-1 (it-PB1) trigonal phase in molten thin films have been studied with transmission electron microscopy, electron diffraction and optical microscopy. The growth rate of trigonal crystals was determined by in situ optical microscopy. It is one hundredth that of $i t$-PB1 tetragonal crystals. The growth rate of trigonal crystals, as well as that of tetragonal crystals, shows the supercooling dependence derived from the nucleation theory. Trigonal crystals grown at 75 and $90.1^{\circ} \mathrm{C}$ possessed well facetted morphology, which suggests the existence of flat growth faces required for nucleation theory between 75 and $90.1^{\circ} \mathrm{C}$. This is consistent with the observed temperature dependence of trigonal crystal growth rate in accordance with the nucleation theory. Lateral surface free energy $\sigma$ of the trigonal phase determined from the observed growth kinetics is about 7 times as large as the value $\sigma^{\text {Hoff }}$ calculated according to Hoffman's equation, while that of the tetragonal phase is roughly in agreement with the estimation. The discrepancy between the values of $\sigma$ and $\sigma^{\text {Hoff }}$ for the trigonal phase can be attributed to the loss of conformational entropy of the ethyl side chains of $i t$-PB1.

KEY WORDS: Isotactic Polybutene-1 / Trigonal Phase / Melt Crystallization / Growth Rate / Self-seeding / Surface Free Energy /
\end{abstract}

Isotactic polybutene-1 (it-PB1) is a semicrystalline polyolefin which exhibits outstanding mechanical properties and pronounced polymorphs that depend on its crystallization conditions. $^{1,2}$ Three different polymorphs, designated trigonal (forms I and $\mathrm{I}^{\prime}$ ) and tetragonal (form II) and orthorhombic phases, have been reported in the literature. ${ }^{3-7}$ Crystallization in the bulk melt under atmospheric pressure yields the tetragonal phase with $11 / 3$ helical chains., ${ }^{3,4}$ The tetragonal phase is unstable. When stored at room temperature, the tetragonal phase undergoes a solid-solid transformation into the trigonal phase with $3 / 1$ helical chains, which is often referred as form I. ${ }^{5}$ The orthorhombic phase has $4 / 1$ helices and is obtained from solution. ${ }^{6}$ Solution crystallization also yields a trigonal phase, and this trigonal phase is usually called form I'. Form I and form $\mathrm{I}^{\prime}$ show the same X-ray and electron diffraction patterns, but have different melting temperatures $\left(130\right.$ vs. $\left.96^{\circ} \mathrm{C}\right) .^{7}$

Despite its excellent mechanical properties, the applications of it-PB1 has been limited considerably by the spontaneous tetragonal-trigonal solid state transformation. The transformation corresponds to a densification and shrinkage of crystals and brings about severe deformation of molded objects. Therefore, bypassing this unstable tetragonal crystallization is of great significance from a practical standpoint.

Numerous attempts have been made to form the trigonal phase in the melt. The earliest challengers were Powers et al., ${ }^{8}$ who used trigonal crystals obtained by solid-state transformation from the tetragonal phase as nuclei and attempted to observe the growth of trigonal crystals in the melt. This was, unfortunately, not successful and they hypothesized that the growth rate of trigonal crystals is "exceedingly" slower than that of tetragonal crystals.

In 1990's, Kopp et al..$^{9}$ reported a breakthrough in forming the trigonal phase. They successfully crystallized the trigonal phase in the melt via epitaxy on aromatic acids or salts, and demonstrated that the trigonal phase can grow in the melt even under atmospheric pressure if appropriate substrates are provided. At an elevated temperature of $110^{\circ} \mathrm{C}$, Zhang et al. found that trigonal crystals can be obtained from molten ultrathin films under atmospheric pressure. ${ }^{10}$

We presented another solution to this issue in our previous works. ${ }^{11-14}$ Using solution-grown trigonal crystals as nuclei, we demonstrated that the trigonal phase can grow in the melt via self-seeding at atmospheric pressure. The self-seeding crystallization does not require the aid of epitaxy and can be applied to crystallization at lower temperatures, which can be an advantage from the standpoint of bulk melt molding. In this paper we report the growth kinetics and morphology of lateral growth shape of melt crystallized it-PB1 trigonal crystals formed by self-seeding.

\section{EXPERIMENTAL}

The $i t$-PB1 used in this study was purchased from Scientific Polymer Products $\left(M_{\mathrm{w}}=185,000\right.$; the melt index is $20 \mathrm{~g} /$ $10 \mathrm{~min}$ ). Thin it-PB1 films were prepared by casting a $0.1 \mathrm{wt} \%$ $p$-xylene solution onto carbon-coated mica kept at $60^{\circ} \mathrm{C}$ on a hot plate. The films were dried in air, an appropriate film thickness of $c a$. $80 \mathrm{~nm}$ being judged by a gold interference color.

\footnotetext{
Department of Bioscience and Bioinformatics, Ritsumeikan University, 1-1-1 Noji-higashi, Kusatsu 525-8577, Japan

*To whom correspondence should be addressed (Present address: Department of Pharmacy, Ritsumeikan University, Tel: +81-77-561-2658, Ext. 8427, Fax: +81-(0)77-561-2659, E-mail: motoi-y@fc.ritsumei.ac.jp).
} 
Crystallization was carried out on a hot stage (Mettler FP82). The $i t$-PB1 films were heated at $128-135^{\circ} \mathrm{C}$ for $2 \mathrm{~min}$ and cooled to a crystallization temperature between $65^{\circ} \mathrm{C}$ and $90.1^{\circ} \mathrm{C}$ at a rate of $15 \mathrm{~K} \mathrm{~min}^{-1}$. In situ observations of the crystallization process were performed using an optical microscope (OM; Nikon OPTIPHOT2); the growth rate was determined from the time dependence of the radius or the major axis of crystals observed by OM.

Transmission electron microscopy (TEM; JEOL JEM1200EXII) was used to identify crystal structures. Samples were examined immediately after crystallization and quenching. The it-PB1-carbon films were floated on a water surface, picked up on electron microscope grids and used as samples.

\section{RESULTS}

Figure 1 shows an $\mathrm{OM}$ image of an $i t$-PB1 thin film that was heated at $132{ }^{\circ} \mathrm{C}$ for $2 \mathrm{~min}$ and then isothermally crystallized at $75^{\circ} \mathrm{C}$. Planar flat-on crystals with somewhat hexagonal morphology are observed. These crystals are encapsulated within a much larger spherulite of the tetragonal phase, which has already been reported to grow from the melt in the literature. ${ }^{15-18}$

When the above crystallized sample was heated again, the flat-on crystals retained their shape up to $124^{\circ} \mathrm{C}$; the surrounding tetragonal spherulites melted below $110^{\circ} \mathrm{C}$, which is consistent with the melting temperature of the tetragonal crystals we reported in our previous work. ${ }^{15}$ The morphology and melting behavior indicate that the flat-on crystals are in a different phase from the surrounding tetragonal crystals.

We confirmed by TEM that the crystals are in the trigonal form. Figure 2 shows a transmission electron micrograph of an it-PB1 film that was heat-treated at $132^{\circ} \mathrm{C}$ for $2 \mathrm{~min}$ and then isothermally crystallized at $75^{\circ} \mathrm{C}$, and its corresponding electron diffraction pattern. A somewhat hexagonally shaped crystal similar to those observed in the OM image can be seen. The electron diffraction pattern shows a net pattern with hexagonal symmetry, and all the Bragg reflections could be indexed with the trigonal form of it-PB1. The melting

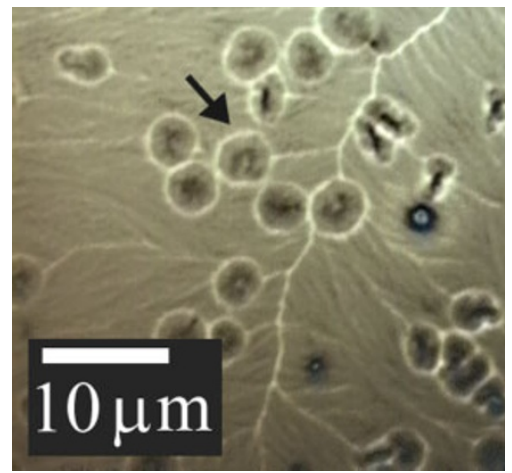

Figure 1. Optical micrograph of it-PB1 trigonal crystals grown at $75^{\circ} \mathrm{C}$. The trigonal crystals are surrounded by two large tetragonal spherulites. The crystal indicated by the arrow shows a somewhat hexagonal morphology.


Figure 2. (a) Electron micrograph and (b) its corresponding selected area electron diffraction pattern of an it-PB1 single flat-on crystal in the trigonal form grown at $75^{\circ} \mathrm{C}$. A flat-on it-PB1 crystal is indicated by an arrow. The arc in the upper part of (a) is an area-selecting aperture.

temperature of the flat-on trigonal crystals observed by $\mathrm{OM}$ is around $124^{\circ} \mathrm{C}$, which is much higher than the melting point of form $\mathrm{I}^{\prime}, 96^{\circ} \mathrm{C}$, and is rather close to that of form I reported by Nakafuku et al., $130^{\circ} \mathrm{C} .{ }^{7} \mathrm{OM}$ and TEM observations indicate that the hexagonally shaped crystals are characterized as form I trigonal crystals grown in the melt.

As mentioned above, trigonal crystals are known to be obtained in solutions. The it-PB1 films used in this study are prepared by casting an it-PB1 solution onto a carbon-coated mica, and therefore contain abundant trigonal crystals grown from the solution. When the it-PB1 films are heated up to a temperature above the equilibrium melting point of the trigonal phase, $136.1^{\circ} \mathrm{C},{ }^{19,20}$ all of the trigonal crystals in the films are completely melted; only the tetragonal phase crystallizes out on cooling to a crystallization temperature. On the contrary, when the films are heated up to a temperature near but below the equilibrium melting point, melting of the trigonal crystals is incomplete, which eventually prepares a number of trigonal nuclei; we can utilize the incompletely melted trigonal crystals for self-seeding. On cooling to a crystallization temperature, we can observe trigonal crystals growing in the melt. These facts are considered to enable the trigonal crystals to grow in the melt even at atmospheric pressure and lower crystallization temperatures.

A sequence of the isothermal crystallization process at $75^{\circ} \mathrm{C}$ after heat-treatment at $132^{\circ} \mathrm{C}$ for $2 \mathrm{~min}$ is shown by successive optical micrographs in Figure 3. Faint circular crystals emerge from the melt in Figure 3a. The crystals slowly increase in size (Figure 3b, 3c) and finally almost all of them impinge upon each other (Figure 3d). A bright region is also observed outside the periphery of each crystal. The bright region is a halo. The widths of these halos are constant during the observation; to obtain the growth rate of the trigonal crystals, we recorded the time dependence of the diameter $2 R$ of each halo as the size of the crystal.

Figure 4 shows an $R$ vs. $t$ plot. The radius $R$ of the flat-on trigonal crystals increased linearly with crystallization time $t$ for all the crystallization temperatures investigated. The linearity indicates that the crystal growth is controlled not by diffusion, but by kinetics of integration of growth units at the interface. 



Figure 3. In situ optical micrographs of it-PB1 trigonal crystals taken at $75^{\circ} \mathrm{C}$ at intervals of $4 \mathrm{~min}$. (a) Faint circular crystals are indicated by the arrows. (b) Halos are observed. (c) The crystals slowly increase in size. (d) Almost all of the crystals impinge upon each other.



Figure 4. Time dependence of radius $R$ of trigonal crystals at several crystallization temperatures.

The growth rate $G_{1}$ was determined from the slope of the time-radius curve. The logarithm of $G_{1}$ is plotted against crystallization temperature in Figure 5. The values of the growth rate $G_{2}$ of tetragonal crystals we observed in our previous work ${ }^{15}$ are also included for comparison. It should be noted that the growth rate of trigonal crystals is one hundredth and one thousandth that of tetragonal crystals around 70 and $90{ }^{\circ} \mathrm{C}$, respectively. This is the reason why the trigonal crystals observed in Figure 1 are surrounded by tetragonal spherulites. The result obtained in this work for the trigonal phase is consistent with the prediction made by Powers et al. in $1965 .{ }^{8}$

The nucleation theory by Hoffman et $a .^{21}$ describes the growth rate $G$ observed at a crystallization temperature $T$ by the following equation:

$$
G=G_{0} \exp \left[-\frac{U}{R\left(T-T_{\mathrm{V}}\right)}\right] \exp \left[-\frac{K}{T \Delta T}\right]
$$

where $G_{0}$ and $K$ are constants, $U$ is the 'activation' energy for polymer diffusion, which is $6280 \mathrm{~J} \mathrm{~mol}^{-122}$ for it-PB1, $R=k N_{\mathrm{A}},\left(k\right.$ is the Boltzmann constant and $N_{\mathrm{A}}$ is Avogadro's

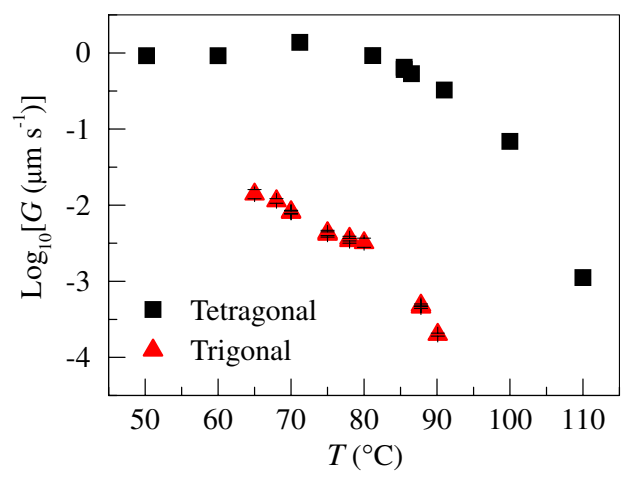

Figure 5. Growth rate $G_{1}$ of trigonal crystals vs. crystallization temperature $T$. Growth rate $G_{2}$ of tetragonal crystals is also included for comparison [ref 15].

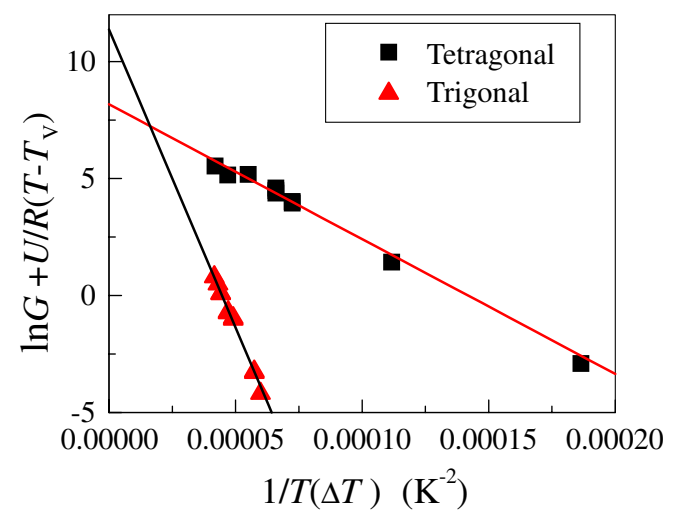

Figure 6. Plot of $\ln G+U / R\left(T-T_{\mathrm{V}}\right)$ vs. $1 / T \Delta T$. Symbols are the same as in Figure 5.

number) $T_{\mathrm{V}}$ is the Vogel temperature $\left(=T_{\mathrm{g}}-30(\mathrm{~K}), T_{\mathrm{g}}\right.$ is the glass transition temperature, which is equal to $-54.2{ }^{\circ} \mathrm{C}^{22}$ for it-PB1), $\Delta T\left(=T_{\mathrm{m}}{ }^{0}-T\right)$ is a supercooling $\left(T_{\mathrm{m}}{ }^{0}\right.$ is the equilibrium melting temperature which takes values of $124.0^{19,20}$ and $136.1^{\circ} \mathrm{C}^{19,20}$ for it-PB1 tetragonal and trigonal phase, respectively, as determined by Gibbs-Thomson analysis in our previous work). The first exponential factor is the VogelFulcher factor for viscosity and the second exponential factor is the surface kinetic factor originally derived assuming flat growth faces. Figure 6 shows $\ln G+U /\left\{R\left(T-T_{\mathrm{V}}\right)\right\}$ as a function of $1 /(T \Delta T)$ for the two phases, using the parameters listed above. The value of $\ln G+U /\left\{R\left(T-T_{\mathrm{V}}\right)\right\}$ depends on $1 /(T \Delta T)$ linearly over the whole range examined for both phases. Eq 1 holds for all the crystallization temperature range investigated; the growth rate of it-PB1 trigonal and tetragonal crystals shows the temperature dependence derived from the nucleation theory.

The extrapolation to zero of the straight lines in Figure 6 for $1 /(T \Delta T)$ gives the values of $G_{0}$ to be $6.33 \times 10^{5} \mu \mathrm{m} \mathrm{s}^{-1}$ and $1.64 \times 10^{4} \mu \mathrm{m} \mathrm{s}^{-1}$ for trigonal and tetragonal crystals, respectively; the slopes give the values of $K$ to be $2.73 \times$ $10^{5} \mathrm{~K}^{2}$ for the trigonal form and $6.46 \times 10^{4} \mathrm{~K}^{2}$ for the tetragonal form. The value of $K$ obtained for the trigonal form is about 4.2 times larger than that obtained for the tetragonal 
(a)

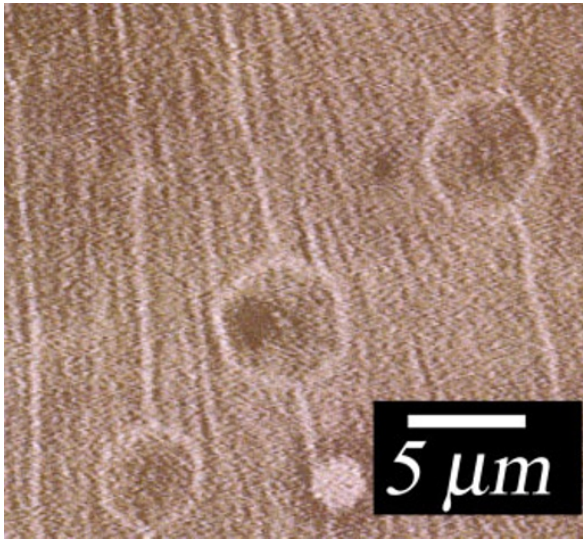

(b)



Figure 7. Lateral morphology of it-PB1 single crystals of the trigonal phase grown at (a) $75^{\circ} \mathrm{C}$ and (b) $90.1^{\circ} \mathrm{C}$ observed by OM. Facetted lateral shapes with a hexagonal morphology are clearly observed both at 75 and $90.1{ }^{\circ} \mathrm{C}$. In Figure $7 \mathrm{a}$, facets are more clearly observed than in Figure 1. This is due to the fact that the trigonal single crystals in Figure $7 \mathrm{a}$ are encapsulated within a large tetragonal single crystal.

form; this indicates that the kinetic barrier for crystallization of the trigonal form is 4.2 times larger than that of the tetragonal form. On the other hand, the value $G_{0}$ of the trigonal form is 39 times larger than that of the tetragonal form.

Figure 7 shows the lateral morphology of single trigonal crystals grown at 90.1 and $75^{\circ} \mathrm{C}$ observed by OM. The observed morphology shows that the trigonal crystals are clearly facetted both at 90.1 and $75^{\circ} \mathrm{C}$. This indicates that trigonal crystals have flat growth fronts between 90.1 and $75^{\circ} \mathrm{C}$. Trigonal crystals are considered to grow by nucleationcontroled growth on their facetted growth fronts. This is consistent with the fact that the temperature dependence of the growth rate of the trigonal crystals follows the nucleation theory.

Assuming that the crystallization is in the regime of multiple nucleation, regime II, as implied from the facetted morphology of growth shape, $K$ in eq 1 is represented by the following expression

$$
K=\frac{2 b \sigma \sigma_{\mathrm{e}} T_{\mathrm{m}}^{0}}{k \Delta h_{\mathrm{f}}}
$$

where $b$ is the layer thickness, which is equal to $0.885 \mathrm{~nm}$ for the trigonal ${ }^{5}$ phase and $0.73 \mathrm{~nm}$ for the tetragonal phase, ${ }^{23} \sigma$ and $\sigma_{\mathrm{e}}$ are the side- and end-surface free energies per unit area, respectively, and $\Delta h_{\mathrm{f}}$ is the heat of fusion per unit volume of a crystal, which takes values of $1.35 \times 10^{8} \mathrm{~J} \mathrm{~m}^{-3}$ for the trigonal form and $1.09 \times 10^{8} \mathrm{~J} \mathrm{~m}^{-3}$ for the tetragonal form. From the values of $K$ and the parameters listed above, the values of $\sigma \sigma_{\mathrm{e}}$ are calculated as $7.04 \times 10^{-4} \mathrm{~J}^{2} \mathrm{~m}^{-4}$ for the trigonal form and $1.68 \times 10^{-4} \mathrm{~J}^{2} \mathrm{~m}^{-4}$ for the tetragonal form. In our previous work, we determined the $\sigma_{\mathrm{e}}$ values to be $4.11 \times 10^{-3} \mathrm{~J} \mathrm{~m}^{-2}$ and $4.36 \times 10^{-3} \mathrm{~J} \mathrm{~m}^{-2}$ for the trigonal and tetragonal phases, respectively. From the values of $\sigma_{\mathrm{e}}$, we obtain the values of $\sigma \equiv \sigma^{\exp }$ to be $17.1 \times 10^{-3} \mathrm{~J} \mathrm{~m}^{-2}$ for the trigonal form and $3.85 \times 10^{-3} \mathrm{~J} \mathrm{~m}^{-2}$ for the tetragonal form (the superscript 'exp' is added to indicate the value is determined experimentally).

\section{DISCUSSION}

In our previous work, we precisely investigated the contribution of the pinning barrier on the basis of numerical calculations reported by Toda, ${ }^{24}$ and we showed that the observed value of $K$ includes not only the contribution of the nucleation barrier, but also the contribution of the pinning barrier. Hence, $K$ is larger than the value of $2 b \sigma \sigma_{\mathrm{e}} T_{\mathrm{m}}^{0} / k \Delta h_{\mathrm{f}}$ and is corrected by including the contribution from the pinning barrier, $\alpha$, as follows ${ }^{15}$

$$
K=\frac{2 b \sigma \sigma_{\mathrm{e}} T_{\mathrm{m}}^{0}}{k \Delta h_{\mathrm{f}}}(1+\alpha)
$$

The value of the lateral surface free energy, $\sigma$, takes a value smaller than the value of $\sigma^{\text {exp }}$, and is corrected by the following equation ${ }^{15}$

$$
\sigma^{\exp }=\sigma(1+\alpha)
$$

where the contribution of the pinning barrier, $\alpha$, is the ratio of the pinning barrier to the nucleation barrier; the expression of $\alpha$ is given as follows ${ }^{15}$

$$
\alpha=\frac{\frac{0.1 k T}{a_{\mathrm{pin}} b_{\mathrm{pin}} \sigma_{\mathrm{e}}}+\frac{0.05 k T}{b_{\mathrm{pin}} c_{\mathrm{pin}} \sigma^{\exp }}}{1-0.05 \frac{k T}{b_{\mathrm{pin}} c_{\mathrm{pin}} \sigma^{\exp }}}
$$

Here, $a_{\text {pin }}, b_{\text {pin }}$ and $c_{\text {pin }}$ are the sizes of growth units as projected along the crystalline $a$-, $b$ - and $c$-axes, respectively, and correspond to the number of monomers that constitute a growth unit. The parameters $a_{\text {pin }}$ and $b_{\text {pin }}$ are the same as the stem parameters $a$ and $b$, respectively. $a_{\text {pin }}=a$ is the width of the stem; $b_{\text {pin }}=b$ is the height of the stem and is equal to the layer thickness. The size of a growth unit means the scale of cooperative motion when polymer chains are incorporated into the crystal phase, and we can naturally consider the $c_{\text {pin }}$ values to be in the range from one turn of helix to persistence length $\left(18\right.$ monomers $^{25}$ ) of $i t$-PB1. The size of a monomer as projected along the $c$-axis is $2.17 \times 10^{-1} \mathrm{~nm}$ for the trigonal phase and $1.93 \times 10^{-1} \mathrm{~nm}$ for the tetragonal phase; when $n$ monomers constitute the growth unit, $c_{\text {pin }}$ of the trigonal and tetragonal phase is expressed as $n \times 2.17 \times 10^{-1} \mathrm{~nm}$ and $n \times 1.93 \times 10^{-1}$ $\mathrm{nm}$, respectively. Figure 8 shows $\sigma$ of the trigonal phase and tetragonal phase calculated from eqs 4 and 5 as functions of the 

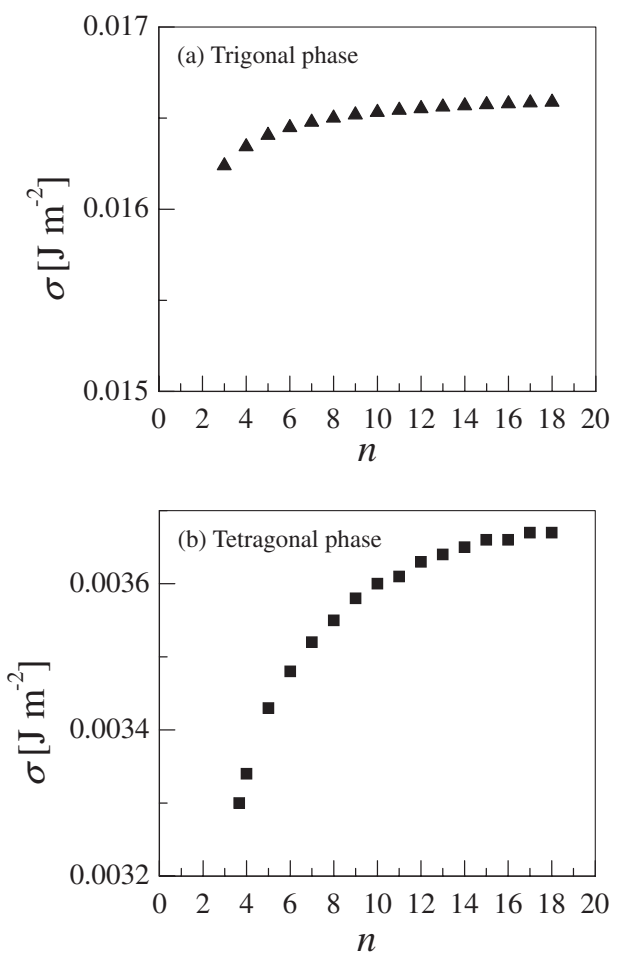

Figure 8. Lateral surface free energy, $\sigma$, of the (a) trigonal phase and (b) tetragonal phase at $85^{\circ} \mathrm{C}$ plotted as functions of the number, $n$, of monomers constituting a growth unit.

number of monomers $n$ constituting a growth unit. For the trigonal phase, one turn of helix is 3 monomers $^{5}$ and $c_{\text {pin }}=$ $6.5 \times 10^{-1} \mathrm{~nm}$; persistence length corresponds to $c_{\text {pin }}=3.9$ nm. We hence obtain the $\sigma$ value to be in the range of $1.62 \times 10^{-2}-1.66 \times 10^{-2} \mathrm{~J} \mathrm{~m}^{-2}$ at $85^{\circ} \mathrm{C}$. For the tetragonal phase, one turn of helix is $11 / 3$ monomers $^{23}$ and $c_{\text {pin }}=$ $7.07 \times 10^{-1} \mathrm{~nm}$; persistence length corresponds to $c_{\text {pin }}=3.47$ $\mathrm{nm}$. The $\sigma$ value is calculated to lie in the range of $3.30 \times$ $10^{-3}-3.67 \times 10^{-3} \mathrm{~J} \mathrm{~m}^{-2}$ at $85^{\circ} \mathrm{C}$.

Next, we will describe a theoretical treatment to estimate the value of the lateral surface free energy. In monatomic systems, Spaepen ${ }^{26}$ proposed that surface free energy should be basically of entropic origin, not of energetic origin. Spaepen showed that surface free energy originates from the loss of configurational entropy which is brought about when liquid adjusts itself to a crystal surface in order to minimize its interfacial energy and density deficits. Liquid atoms at the crystal-liquid interface can not adopt as many configurations as in the bulk because of the special boundary conditions of the crystal plane. This concept was introduced into the systems of $n$-alkanes as the 'negentropic model' by Turnbull and Spaepen. ${ }^{27}$ They found that scaled crystal-melt interfacial tensions fall off asymptotically to a constant value with increasing chain length; they explained this by the segmental nature of $n$-alkane chains. In this case, the adjustment consists of orienting the molecular segments of the melt near the interface so that they are parallel to the crystal boundary plane. In the 1990s, Hoffman et al. ${ }^{21,28,29}$ related the sizes of segmentalized chain molecules to the characteristic ratio $C_{\infty}$ of the rotational isometric state model of the melt state of polymers, and succeeded in expressing the lateral surface free energy $\sigma$ of polymer crystals grown in the melt in a unified manner as follows:

$$
\sigma=T\left(\frac{1}{C_{\infty}} \frac{\Delta h_{\mathrm{f}}}{T_{\mathrm{m}}^{0}}\right)\left(\frac{a}{2}\right)\left(\frac{l_{\mathrm{b}}}{l_{\mathrm{u}}}\right) \equiv \sigma^{\text {Hoff }}
$$

where $a$ is the width of the stem, $l_{\mathrm{b}}$ the bond length, and $l_{\mathrm{u}}$ the $\mathrm{C}$-C distance as projected along the $c$-axis. The conventional nucleation theory explains that the free energy barrier of nucleation is the barrier to building two lateral surfaces of a surface nucleating stem. In the unperturbed melt, polymer chains are segmentalized into roughly straight sections of Kuhn length $C_{\infty} l_{\mathrm{b}}$ on average. In other words, a polymer chain in the melt can be regarded as a sequence of roughly straight segments of Kuhn length. In the derivation of eq 6, Hoffman et al. hypothesized that polymer chains 'segmentalize' into sections of length $C_{\infty} l_{\mathrm{b}}$, which need to be 'aligned' along the growth front before they become crystallographically attached into the crystal phase and turn into nucleating stems. They postulated that the nucleation barrier results from the reduction of conformational entropy required for polymer chains to form the 'segmentalized and aligned' activated state; this shows up as the lateral surface free energy. Since polymer chains are already divided into roughly straight segments of average length $C_{\infty} l_{\mathrm{b}}$ in their unperturbed state, the reduction of entropy that takes place to form the 'segmentalized and aligned' activated state is much smaller than the full entropy of fusion of bulk crystals, which is $\Delta S_{\mathrm{f}} \equiv \Delta h_{\mathrm{f}} / T_{\mathrm{m}}^{0}$ per unit volume. They estimated the reduced entropy of the aligned segments to be $\Delta S_{\mathrm{f}} / C_{\infty}$; this turns up as the second factor in the right hand side of eq 6 . The depiction above explains that the lateral surface free energy originates from the loss of conformational entropy that occurs when polymer chains become 'segmentalized and aligned,' i.e., stretched along the growth face. The detailed derivation is given as follows. When a portion of polymer chain constituted of $m$ backbone $\mathrm{C}$ atoms becomes incorporated into the crystal phase and turns into a nucleating stem, the overall change of entropy as measured relative to the melt is $-\left(\Delta h_{\mathrm{f}} / T_{\mathrm{m}}^{0}\right) a b m l_{\mathrm{b}}$. The reduction of entropy required to pass the 'segmentalized and aligned' activated state is only $1 / C_{\infty}$ of the change and is calculated to be $-\left\{\Delta h_{\mathrm{f}} /\left(T_{\mathrm{m}}^{0} C_{\infty}\right)\right\} a b m l_{\mathrm{b}}$. The increase in free energy of this activated state is $T\left\{\Delta h_{\mathrm{f}} /\left(T_{\mathrm{m}}^{0} C_{\infty}\right)\right\} a b m l_{\mathrm{b}}$; the increased free energy is interpreted as the nucleation barrier and is equal to the work $2 b \sigma m l_{\mathrm{u}}$ needed for building the two lateral surfaces of a nucleating stem. Equating the work $2 b \sigma m l_{\mathrm{u}}$ with the barrier $T\left\{\Delta h_{\mathrm{f}} /\left(T_{\mathrm{m}}^{0} C_{\infty}\right)\right\} a b m l_{\mathrm{b}}$, eq 6 is obtained. Eq 6 has been successful in predicting the values of $\sigma$ for a number of meltcrystallized polymers with simple side chains such as polyethylene (PE), isotactic polypropylene (it-PP), and poly(Llactic acid) (PLLA). ${ }^{28}$

In the case of $i t-\mathrm{PB} 1, l_{\mathrm{b}}$ is $1.54 \times 10^{-1} \mathrm{~nm}$. For the trigonal phase, $l_{\mathrm{u}}$ is $1.08 \times 10^{-1} \mathrm{~nm}$ and $a$ is $5.10 \times 10^{-1} \mathrm{~nm}$; for the tetragonal phase, $l_{\mathrm{u}}$ and $a$ take values of $9.64 \times 10^{-2} \mathrm{~nm}$ and 
$7.30 \times 10^{-1} \mathrm{~nm}$, respectively. Using the parameters listed above and the characteristic ratio $C_{\infty}=18.0$ of it-PB1 reported in the literature, ${ }^{25} \sigma^{\text {Hoff }}$ of the trigonal form is estimated as $2.38 \times 10^{-3} \mathrm{~J} \mathrm{~m}^{-2}$ at $85^{\circ} \mathrm{C}$ from eq 6 . This estimation is about one seventh the value of $\sigma$ of the trigonal form obtained above from experiments, and eq 6 does not hold. On the other hand, $\sigma^{\text {Hoff }}$ of the tetragonal form at $85^{\circ} \mathrm{C}$ is estimated as $3.19 \times 10^{-3} \mathrm{~J} \mathrm{~m}^{-2}$, which is roughly in agreement with the experimentally obtained value of $\sigma=3.30 \times 10^{-3}$ $3.67 \times 10^{-3} \mathrm{~J} \mathrm{~m}^{-2}$. It is of great interest that eq 6 does not hold for trigonal crystals while it roughly holds for tetragonal crystals growing in the same environment.

The discrepancy between the values of $\sigma$ and $\sigma^{\text {Hoff }}$ for the trigonal form can be attributed to the loss of conformational entropy of $i t$-PB1 side chains; when an it-PB1 chain forms a nucleating stem of the trigonal form, it is assumed that the chain needs not only to become "segmentalized and aligned," but also to have its side chains "fixed" in the crystallographically correct conformation. The restricted conformation of the side chains generates an excessive amount of free energy barrier due to the loss of conformational entropy of the side chains; this can account for the much larger value of lateral surface free energy $\sigma$ than the value of $\sigma^{\text {Hoff }}$ estimated from eq 6. If we assume that the ethyl side groups in the segmentalized-aligned it-PB1 chains should adopt the conformation which is the same as that inside the trigonal crystal phase, the loss of conformational entropy of a side chain in a monomer is roughly estimated as $k \ln 3$, since the ethyl side group of it-PB1 is an articulated side chain and the three rotational isometric states can clearly be distinguished. The increase of free energy barrier of nucleation per monomer due to the loss of conformational entropy of a side chain, $\Delta f_{\text {side, }}$, is written as:

$$
\Delta f_{\text {side }} \cong\left(1-\frac{1}{C_{\infty}}\right) k T \ln 3
$$

where the term $\left(-1 / C_{\infty}\right)$ in the first factor of the right hand side is added to subtract the free energy barrier already counted in eq 6 , because the full entropy of fusion of both the side chains and main chains multiplied by a factor $1 / C_{\infty}$ is considered to be the free energy barrier of forming a nucleating stem in the derivation of eq 6 . The value of $\Delta f_{\text {side }}$ is thus calculated to be $5.13 \times 10^{-21} \mathrm{~J}$ at $85^{\circ} \mathrm{C}$. On the other hand, the observed excessive amount of free energy barrier of nucleation per monomer, $\Delta \phi_{\mathrm{ex}}$, is represented by:

$$
\Delta \phi_{\mathrm{ex}}=2 l_{\mathrm{m}} b\left(\sigma-\sigma^{\mathrm{Hoff}}\right)
$$

where $l_{\mathrm{m}}$ is the size of the monomer as projected along the $c$-axis and is equal to $2.17 \times 10^{-1} \mathrm{~nm}$ for the trigonal form. Eq 8 yields the value of $\Delta \phi_{\text {ex }}$ to be $5.31 \times 10^{-21}-5.45 \times$ $10^{-21} \mathrm{~J}$ for the trigonal form at $85^{\circ} \mathrm{C}$. This is roughly in agreement with the value of $\Delta f_{\text {side. }}$. The agreement indicates that the difference between the values of $\sigma$ and $\sigma^{\text {Hoff }}$ can be attributed to the loss of entropy caused by the conformational restriction of the side groups. We hence obtain the expression of $\sigma$ of the trigonal phase related to $\sigma^{\text {Hoff }}$ and $\Delta f_{\text {side }}$ as follows

$$
\sigma=\sigma^{\text {Hoff }}+\frac{\Delta f_{\text {side }}}{2 l_{\mathrm{m}} b}
$$

For the tetragonal form, $\Delta \phi_{\mathrm{ex}}$ is calculated to be $0.31 \times 10^{-22}-1.36 \times 10^{-22} \mathrm{~J}$, which is much smaller than that of the trigonal form. This is interpreted as follows: when an itPB1 chain forms a nucleating stem in the tetragonal phase, on the contrary, the loss of conformational entropy of the side chains is much less effective. Maring et al. ${ }^{30}$ showed that the itPB1 chains in the tetragonal phase are mobile and have disordered conformation, while those in the trigonal phase are ordered and well rigid by NMR. Moreover, Miyoshi et al. ${ }^{31,32}$ reported that the side chains of the tetragonal phase are disordered and highly mobile from NMR. Due to the dynamic conformational disorder of the side chains of the tetragonal crystalline phase, side chains are considered to be imposed on much less conformational restriction; the loss of conformational entropy for the side chains to nucleate is much smaller and the 'segmentalized and aligned' state is the main part of nucleation barrier. This can account for the fact that eq 6 roughly holds for the tetragonal phase. (The trigonal phase is reported to have a statistically disordered structure. ${ }^{5}$ At one site of the lattice, upward and downward chains of the same helical handedness are statistically located at a weight of $50 \%$. However, this does not mean side chains are disordered within each helix. Side chains have ordered conformation within each helix, which, we consider, makes the barrier of nucleation in the trigonal phase larger than that of the trigonal phase.)

The difference between the growth kinetics of the two crystal forms is considered to consist in the conformational entropy of the ethyl side groups of it-PB1, and this results in the disablement of Hoffman's equation for the trigonal form. Since it is mainly side chains that are exposed to the crystalmelt interface, the concept that the loss of conformational entropy of side chains contributes to the barrier of nucleation is also naturally supported by the original 'negentropic model,' in which liquid molecules at the crystal-liquid interface adjust themselves to the crystal plane, reducing their configurational entropy. The trigonal form has larger density $\rho$ than the tetragonal form $\left(0.95 \mathrm{~g} \mathrm{~cm}^{-3} v s .0 .888 \mathrm{~g} \mathrm{~cm}^{-3}\right.$; for the amorphous phase, $\left.\rho=0.87 \mathrm{~g} \mathrm{~cm}^{-3}\right)$, and has larger crystal-liquid density difference. In the incorporation of it-PB1 chains into the trigonal phase, the ethyl side chains need to adjust themselves into more ordered conformation close to that in the crystal phase so that they can minimize the interfacial energy and density deficit.

The model developed in this study can also be supported from the standpoint of crystal structures as follows. In the trigonal phase, tight $3 / 1$ helical chains are packed within hexagonal lattice as shown in Figure 9a. Within each helical repeating unit composed of three monomers, a helical chain is in contact with a neighbouring chain with two ethyl side chains and every side chain is involved in inter-helix contact. Ethyl side chains in neighbouring helices are displaced half the lattice constant along the $c$-axis, and a side chain is in contact with two side chains in a neighbouring helical chain, as shown 


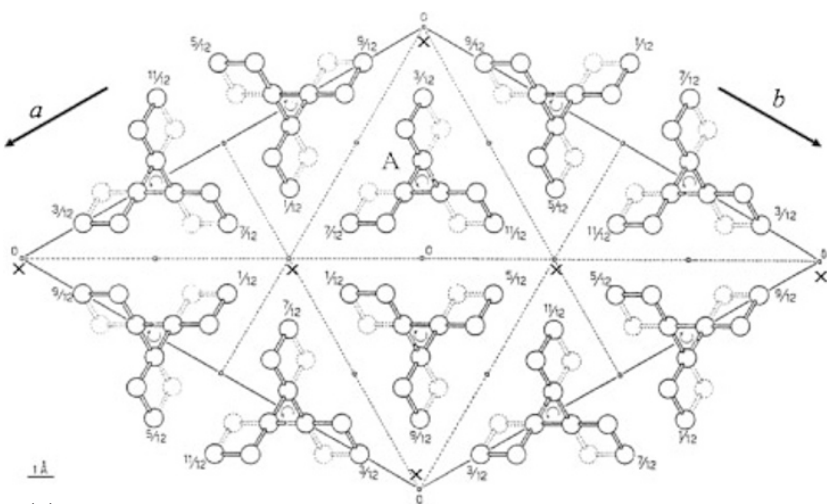

(a)
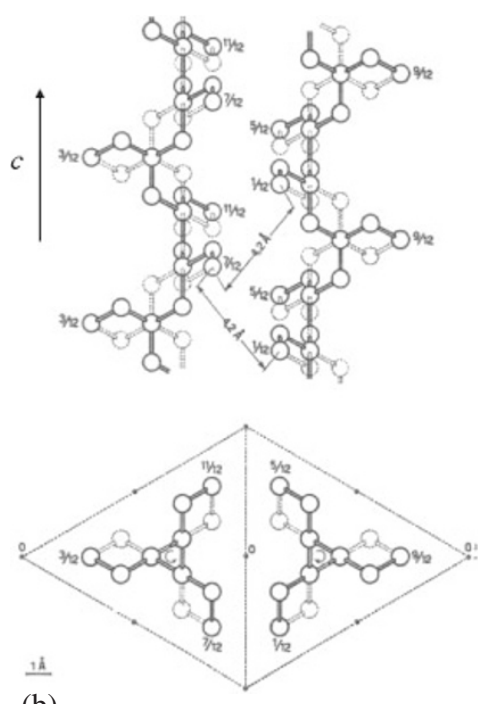

(b)

Figure 9. (a) Crystal structure of it-PB1 trigonal phase projected on $a b$ plane. Only $\mathrm{C}$ atoms are shown. Fractional coordinates of carbon atoms at the ends of ethyl side chains along the $c$-axis are shown. The dotted lines present positions of chains generated by statistical disorder. (b) Projection on $a b$ plane and on a plane parallel to the $c$-axis of two contacting helices in the it-PB1 trigonal phase. (Figure 9a, 9b were taken from ref 5.)

in Figure 9b. Since each side chain is in contact with two helices as shown in Figure 9a, each side chain is mutually locked by four neighbouring side chains, which prevents the rotation of side chains. Moreover, six helices gathers at each points marked with " $x$ " in Figure 9a; this makes six helices locked up by each other. These make it substantially impossible for ethyl side chains to have conformational disorder. When it-PB1 chains directly crystallize from the melt, each of the two out of three side chains in a helical repeating unit need to be infixed between two side chains on the crystal (110) plane. (For example, in the deposition of the helix marked with "A" in Figure 9a onto (110) plane, two side chains of fractional coordinate $7 / 12$ and $11 / 12$ need to be infixed.) Hence chains can not attain crystallographic incorporation into the trigonal phase without removing conformational disorder of ethyl side chains. The conventional "segmentalization and alignment" of the main chains is considered to be insufficient as the activated state for crystallographic incorpo-
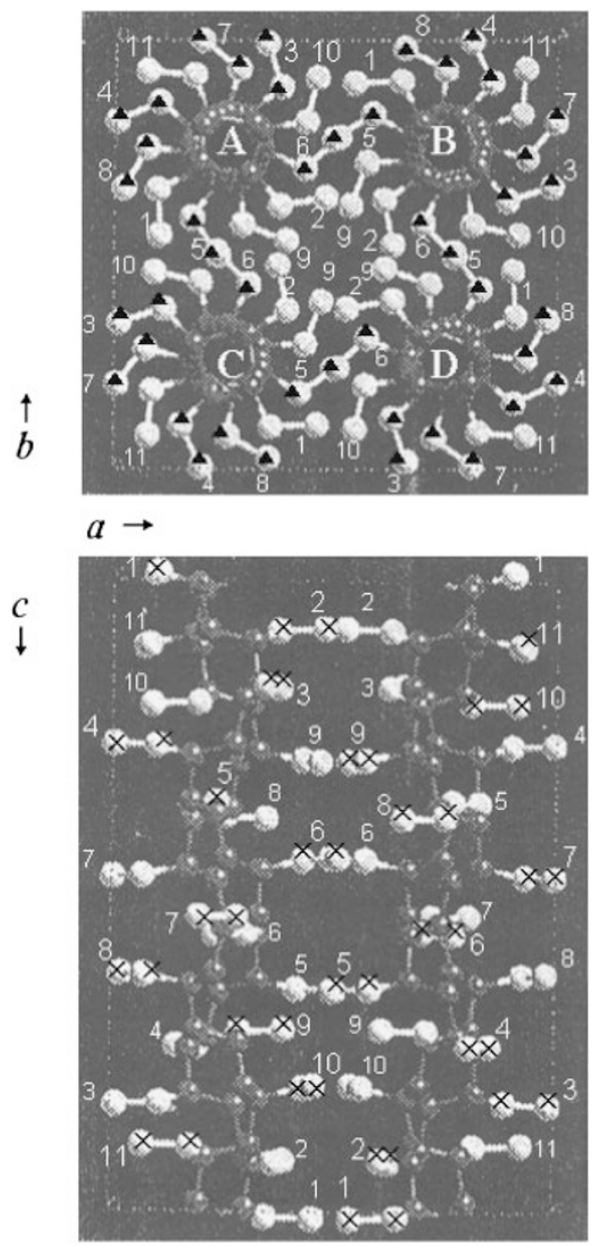

Figure 10. Crystal structure of it-PB1 tetragonal phase. (Taken from ref 23.) Only $C$ atoms are shown. The four helices in the unit cell is marked with alphabets from A to D. Side chains in each helix is numbered from 1 to 11 so that we can distinguish each of the 44 side chains in the unit cell. For example, the side chain numbered " 3 " in the helix A can be denoted by "A3." Among 44 side chains in the unit cell, only pairs of A6-B5, A5-C6, C5-D6, B6-D5, A4-B7, A8-B3, A7-C4, A3-C8, C3-D8, C7-D4, B8-D3 and B4-D7 are involved in interlocking to neighbouring helices. Other side chains are weakly contacted. For example, side-chain pairs of A3-B9, A9-B5 and C9-B9 seems to be closely in contact, respectively, seen along the $b$-axis. However, it is clear they are distantly positioned, seen along the $c$-axis.

ration of it-PB1 chains; side chains need to take crystallographically correct conformation. This shows up as the $\sigma$ value of the trigonal phase excessively larger than that evaluated by eq 6 . In the tetragonal phase, on the other hand, loose $11 / 3$ helices are packed within tetragonal lattice (Figure 10). Within each helical repeating unit composed of 11 monomers, a helical chain is interlocked with its four neighbouring chains via six side chains. (Interlocking side chains are marked with filled triangle on the projection onto $a b$ plane in Figure 10.) One side chain is involved for the contact with each of the two helices within the unit cell; two side chains are involved for the contact with each of the two helices outside the unit cell. The rest five side chains are weakly in contact with the neighbouring chains. These weakly contacted side chains are considered to be loosely bounded in their conformation. Moreover, vacant space 
is observed to exist amid the four helices seen along the c-axis. The vacant space is adjacent to the weakly contacted five side chains. Conformational disorder requires extra volume in the neighbourhood. The vacant space can provide the loosely bounded ethyl side chains with extra volume necessary for them to be conformationally disordered. These factors are considered to enable ethyl side chains in the tetragonal phase to have abundant conformational disorder, and to help the "segmentalized-aligned chains" to have conformational disorder. When an it-PB1 chain becomes attached on the crystal (100) plane of the tetragonal phase and forms a nucleating stem, only one or two out of 11 side-chains in the helical repeating unit need to be fixed. Even if the other side chains have excessive conformational disorder, it is envisioned that the excessive defects can be repaired after crystallographic attachment. Hence, it-PB1 chains are considered to attain crystallographic incorporation into the tetragonal phase with many of the side chains having conformational disorder in the "segmentalized-aligned" activated state. This means that the conventional "segmentalization and alignment" of main chains is almost sufficient for crystallographic incorporation, which shows up as the $\sigma$ value in accordance with the evaluation by eq 6 .

In polymer systems for which eq 6 has been successful, the side chains of the polymers are simple and therefore the loss of conformational entropy of the side chains is zero or almost negligible. The methyl side groups of it-PP and PLLA, for example, have three-fold symmetry and none of the three rotational isometric states can be distinguished from the other two; PE has no side chains and has no conformational entropy of side chains. In such systems, the conformational entropy of side chains is negligible. For these polymers, the reduction in conformational entropy in the 'segmentalized and aligned' state is identical to the loss of entropy needed for the main chains to become stretched; $\sigma$ is therefore determined by the reduction of conformational entropy caused in the 'segmentalized and aligned' process of the main chains assumed in the original 'negentropic model'. This is why eq 6 holds very well for these polymers. On the other hand, the articulated ethyl side chains of $i t$-PB1 have conformational entropy due to the three distinguishable rotational isometric states. We hence need to introduce another 'negentropic' process that limits the conformation of side chains when the 'segmentalized and aligned' $i t$-PB1 chains become incorporated into the trigonal crystal phase. By taking the conformational entropy of side chains into account, we can resume the validity of Hoffman's equation and expect to extend its applicability to polymers with larger and complicated side chains.

The trigonal phase of it-PB1 is the first experimentally demonstrated example of polymer crystal whose lateral surface free energy $\sigma$ clearly deviates from Hoffman's equation. In order to prove our hypothesis, we need to investigate large numbers of polymers with different side and main chains. More precise and systematic investigations are deemed necessary that deals with the size and symmetry of side chains. A research is already underway on poly-4-methyl-1-pentene and poly-3-methyl-1-butene, which have bulky isobutyl and isopropyl side chains.

\section{CONCLUSION}

The growth rate of $i t$-PB1 trigonal crystals was determined by in situ $\mathrm{OM}$ in the melt. The observed growth rate of the trigonal crystals is one hundredth that of the tetragonal crystals around $75^{\circ} \mathrm{C}$. The growth rate of it-PB1 trigonal crystals, as well as that of tetragonal crystals, shows the temperature dependence derived from the nucleation theory.

Trigonal crystals grown at 75 and $90.1{ }^{\circ} \mathrm{C}$ possessed well facetted morphology. Flat growth faces required for the nucleation theory are considered to exist between 75 and $90.1^{\circ} \mathrm{C}$. This supports the observed temperature dependence of trigonal crystal growth rate in accordance with the nucleation theory.

Lateral surface free energies $\sigma$ of the trigonal and tetragonal phases were determined to be in the range of $1.62 \times 10^{-2}$ $1.66 \times 10^{-2} \mathrm{~J} \mathrm{~m}^{-2}$ and $3.30 \times 10^{-3}-3.67 \times 10^{-3} \mathrm{~J} \mathrm{~m}^{-2}$, respectively, with the contribution of the pinning barrier taken into consideration. The $\sigma$ value of the trigonal phase is larger than the value $\sigma^{\text {Hoff }}=2.38 \times 10^{-3} \mathrm{~J} \mathrm{~m}^{-2}$ estimated from the theory by a factor of about 7 , while the value $\sigma$ of the tetragonal phase is in good agreement with the value of $\sigma^{\text {Hoff }}=3.19 \times 10^{-3} \mathrm{~J} \mathrm{~m}^{-2}$. This means that Hoffman's equation of lateral surface free energy does not hold for the trigonal form, while it roughly holds for the tetragonal form. The disablement of Hoffman's equation for the trigonal form is considered to be caused by the conformational entropy of the ethyl side chains of it-PB1. When an it-PB1 chain forms a nucleating stem of the trigonal phase, the chain needs not only to become "segmentalized and aligned," but also to have its side chains restricted in the crystallographically correct conformation. The restricted conformation of the side chains generates an excessively large free energy barrier due to the loss of conformational entropy of the side chains, leading to a value of $\sigma$ that is much larger than the theoretical estimation.

Acknowledgment. This work was partially supported by "Academic Frontier" Project from MEXT, 2004-2008. The author MY expresses his sincere thanks Professor Toda (Hiroshima University), Professor Miyaji (Kyoto University) and Professor Fukao (Ritsumeikan University) for valuable discussions and encouragement.

Received: October 31, 2007

Accepted: June 23, 2008

Published: August 22, 2008

\section{REFERENCES}

1. R. L. Miller and V. F. Holland, J. Polym. Sci., Part C: Polym. Lett., 2, 519 (1964).

2. F. Danusso and G. Gianotti, Macromol. Chem., 61, 139 (1963).

3. A. Turner-Jones, Polymer, 7, 23 (1976).

4. A. Turner-Jones, J. Polym. Sci., Part C: Polym. Lett., 1, 455 (1963). 
5. G. Natta, P. Corradini, and I. W. Bassi, Nuovo Cimento Suppl., 15, 52 (1960).

6. V. F. Holland and R. L. Miller, J. Appl. Phys., 35, 3241 (1964).

7. C. Nakafuku and T. Miyaki, Polymer, 24, 141 (1984).

8. J. Powers, J. D. Hoffman, J. J. Weeks, and F. A. Quinn Jr., J. Res. Natl. Bur. Stand. (U.S.), 69A, 335 (1965).

9. S. Kopp, J. C. Wittmann, and B. Lotz, Polymer, 35, 916 (1994).

10. B. Zhang, D. Yang, and S. Yan, J. Polym. Sci., Part B: Polym. Phys., 40, 2641 (2002).

11. M. Yamashita, A. Hoshino, and M. Kato, J. Polym. Sci., Polym. Phys. Ed., 45, 684 (2007).

12. M. Yamashita, J. Cryst. Growth., 310, 1739 (2008).

13. M. Yamashita and T. Takahashi, Kobunshi Ronbunshu, 65, 218 (2008).

14. M. Yamashita and S. Ueno, Cryst. Res. Technol., 42, 1222 (2007).

15. M. Yamashita, H. Miyaji, K. Izumi, and A. Hoshino, Polym. J., 36, 226 (2004).

16. H. Miyaji, Y. Miyamoto, K. Taguchi, A. Hoshino, M. Yamashita, O. Sawanobori, and A. Toda, J. Macromol. Sci., B42, 867 (2003).

17. M. Yamashita and T. Takahashi, in "Modern Research and Educational Topics in Microscopy," A. Méndez-Vilas, Ed., Formatex Research Center, Badajoz, Spain, 2007, Vol. 2, p 713.

18. M. Yamashita and M. Kato, J. Appl. Crystallogr., 40, s650 (2007).

19. U. Leute and W. Dollhopf, Colloid Polym. Sci., 261, 299 (1983).
20. M. Yamashita and M. Kato, J. Appl. Crystallogr., 40, s558 (2007).

21. J. D. Hoffman and R. L. Miller, Polymer, 38, 3151 (1997).

22. J. D. Hoffman, G. T. Davis, and J. I. Lauritzen Jr., in "Treatise on Solid State Chemistry,” N. B. Hannay, Ed., Plenum, New York, 1976, Chap. 7, p. 497.

23. K. Tashiro, A. Saiani, S. Miyashita, Y. Chatani, and H. Tadokoro, Polym. Prepr., Jpn., 47, 3869 (1998).

24. A. Toda, J. Chem. Phys., 118, 8446 (2003).

25. M. Kurata and Y. Tsunashima, in "Polymer Handbook" 4th ed., J. Brandrup, E. H. Immergut, and E. A. Grulke, Ed., Interscience Publishers, New York, 1999, Chap. 6, pp VI/48-49.

26. S. Spaepen, Acta. Metall. Mater., 23, 729 (1975).

27. D. Turnbull and F. Spaepen, J. Polym. Sci., Polym. Symp., 63, 237 (1963).

28. J. D. Hoffman, Polymer, 33, 2643 (1992).

29. J. D. Hoffman, R. L. Miller, H. Marrand, and D. B. Roitman, Macromolcules, 25, 2221 (1992).

30. D. Maring, B. Meurer, and G. Weill, J. Polym. Sci., Part B: Polym. Phys., 33, 1235 (1995).

31. T. Miyoshi, S. Hayashi, F. Imashiro, and A. Kaito, Macromolecules, 35, 6060 (2002).

32. T. Miyoshi, S. Hayashi, F. Imashiro, and A. Kaito, Macromolecules, 35, 2624 (2002). 